\title{
Approach to Equilibrium in Weakly Coupled Non-Abelian Plasmas
}

\author{
Aleksi Kurkela ${ }^{1}$ and Egang $\mathrm{Lu}^{2}$ \\ ${ }^{1}$ Physics Department, Theory Unit, CERN, CH-1211 Genève 23, Switzerland \\ ${ }^{2}$ Department of Physics, McGill University, 3600 rue University, Montréal, Quebec H3A 2T8, Canada
}

(Received 6 June 2014; published 28 October 2014)

\begin{abstract}
We follow the time evolution of non-Abelian gauge bosons from far-from-equilibrium initial conditions to thermal equilibrium by numerically solving an effective kinetic equation that becomes accurate in the weak coupling limit. We consider isotropic initial conditions that are either highly overoccupied or underoccupied. We find that overoccupied systems thermalize through a self-similar cascade reaching equilibrium in multiples of a thermalization time $t_{\mathrm{eq}} \approx 72 . /\left(1+0.12 \log \lambda^{-1}\right) \times 1 / \lambda^{2} T$, whereas underoccupied systems undergo a "bottom-up" thermalization in a time $t_{\mathrm{eq}} \approx[34 .+21 . \log (Q / T)] /$ $\left(1+0.037 \log \lambda^{-1}\right) \times(Q / T)^{1 / 2} / \lambda^{2} T$, where $Q$ is the characteristic momentum scale of the initial condition. We apply this result to model initial stages of heavy-ion collisions and find rapid thermalization roughly in a time $Q t_{\text {eq }} \lesssim 10$ or $t_{\text {eq }} \lesssim 1 \mathrm{fm} / c$.
\end{abstract}

Non-Abelian far-from-equilibrium plasmas occur in many cosmological pre- or reheating scenarios [1] or due to possible cosmological phase transitions [2], as well as in the early stages of heavy-ion collisions. These far-from-equilibrium systems may be overoccupied, such that the energy is spread out in longer wavelength modes than in thermal equilibrium but with stronger fields. This is the case, e.g., for fields generated through parametric resonance, and in heavy-ion collisions, at least in the limit of asymptotically large center of mass energies, where the initial condition may be described by using the colorglass-condensate framework [3]. Alternately, far-fromequilibrium systems may be underoccupied, such that the system consists of fewer, but more energetic, quasiparticles than the corresponding thermal system. This is the case in, e.g., Planck-suppressed decay of inflatons [4]. Also, even though the initial condition of heavy-ion collisions is overoccupied, it has been demonstrated by Baier, Mueller, Schiff, and Son [5] (see also [6]) that the longitudinal expansion renders the prethermal fireball underoccupied before it reaches local thermal equilibrium.

This has motivated several numerical [7-12] and analytical $[13,14]$ works to study simple far-from-equilibrium model systems, in particular, that of a single species of gauge bosons in a (nonexpanding) flat space-time with statistically isotropic initial conditions at weak coupling, which we investigate in this Letter with both over- and underoccupied initial conditions. In both cases, we follow the time evolution of the system from the initial far-fromequilibrium state to thermal equilibrium and extract the thermalization time, which we define as the exponent governing relaxation of the deviation of the first moment of the distribution function, $\langle|p|\rangle=\int_{\mathbf{p}}|p| f(p) / \int_{\mathbf{p}} f(p)$, from its equilibrium value $\langle|p|\rangle_{T}$ at late times:

$$
\langle|p|(t)\rangle-\langle|p|\rangle_{T} \propto e^{-t / t_{\mathrm{eq}}}
$$

In the overoccupied case, early dynamics fall onto a nonthermal attractor solution: if the initial momentum scale characterized by $Q^{2} \equiv\left\langle p^{2}(t=0)\right\rangle$ is much smaller than the momentum scale of the target thermal system, then the scattering time of the initial system $\tau_{\text {init }} \sim(Q / T)^{7} /\left(\lambda^{2} T\right)$ [13] is faster than that of the thermalized system $\tau_{\text {therm }} \sim 1 /\left(\lambda^{2} T\right)$, with $T$ the final equilibrium temperature and $\lambda \equiv N_{c} g^{2}$ the 't Hooft coupling. While it takes the system at least $\tau_{\text {therm }}$ to reach thermal equilibrium, it takes only $\tau_{\text {init }} \ll \tau_{\text {therm }}$ to lose detailed information of its initial condition. Therefore, at times $\tau_{\text {init }}<t<\tau_{\text {therm }}$ the system will reside in a state that is almost independent of the initial condition but time varying; this is the nonthermal fixed point. The fixed point is described by a scaling form of the occupation function $f(p, t)=t^{-4 / 7} \tilde{f}\left(p / p_{\max }\right)$, where the scaling function $\tilde{f}$ is proportional to $1 / p$ at small momenta and decays exponentially above an evolving scale $p_{\max } \propto$ $Q(Q t)^{1 / 7}[12,13]$. There have been several numerical studies demonstrating the approach to and details of the fixed point using the classical Yang-Mills theory [7-11] and the effective kinetic theory (EKT) of Arnold, Moore, and Yaffe [12,15]. (For a discussion of the sensitivity of the initial conditions and how the attractor is reached, see, in particular, [10].) The simulations have so far been limited to the classical limit $(f \gg 1$ and $\lambda \ll 1)$, and therefore the transition from the nonthermal fixed point to thermal equilibrium (with $f \sim 1$ ) has not been addressed and current estimates of the thermalization time are purely parametric. In this Letter, we demonstrate using numerical simulations of EKT that the fixed point solution-and any initial condition that falls to the basin of attraction of the fixed point-reaches thermal equilibrium in a time proportional to 


$$
t_{\mathrm{eq}}^{\text {overocc }} \approx \frac{72 .}{1+0.12 \log \lambda^{-1}} \frac{1}{\lambda^{2} T} .
$$

In the underoccupied case, the scattering time of the initial state is much longer than that of the target thermal system, and therefore the system loses information about the initial conditions only on final thermalization. The equilibration of the underoccupied system proceeds via bottom-up thermalization $[5,13]$ : the initial hard particles radiate soft radiation, which forms a thermal background through which the hard particles propagate. Interaction with the thermal background eventually causes the hard particles to undergo a radiational breakup releasing their energy to the thermal bath. (Within this definition, the bottom-up thermalization is not specific to expanding systems but refers to the order at which modes at different momentum scales thermalize.) Parametrically, the breakup takes place in the same time scale that it takes for a jet of momentum $Q$ to be quenched (i.e., to lose most of its energy) in a thermal bath of temperature $T$ : $\tau_{\text {eq }} \sim 1 / \lambda^{2} T(Q / T)^{1 / 2}[16,17]$. The dominant interaction is between the hard particles and the thermal bath, not among the hard particles themselves [5,13], and therefore the equilibration depends only on averaged features of the initial condition. Indeed, we find that we can express the thermalization time to good accuracy as a function of the initial momentum scale $Q$ only:

$$
t_{\mathrm{eq}}^{\text {underocc }} \approx \frac{34 .+21 \cdot \log (Q / T)}{1+0.037 \log \lambda^{-1}}\left(\frac{Q}{T}\right)^{1 / 2} \frac{1}{\lambda^{2} T} .
$$

Effective kinetic theory.-In the weak coupling limit $\lambda \rightarrow 0$, the evolution of modes with perturbative occupancies $\lambda f(p) \rightarrow 0$ and whose momenta are larger than the screening scale $p^{2} \gg m^{2} \equiv 2 \lambda \int_{\mathbf{p}} f(p) /|p|$ can be described to leading order in $\lambda f$ by an effective kinetic equation for the color-averaged gauge boson distribution function [15]

$$
\partial_{t} f(p, t)=-\mathcal{C}_{2 \leftrightarrow 2}[f](p)-\mathcal{C}_{1 \leftrightarrow 2}[f](p)
$$

with

$$
\begin{aligned}
\mathcal{C}_{2 \leftrightarrow 2}[f](p)= & \frac{1}{2} \int_{\mathbf{k}, \mathbf{p}^{\prime}, \mathbf{k}^{\prime}} \frac{|\mathcal{M}(m)|^{2}(2 \pi)^{4} \delta^{(4)}\left(p+k-p^{\prime}-k^{\prime}\right)}{2 k 2 k^{\prime} 2 p 2 p^{\prime}}\left\{f_{p} f_{k}\left[1+f_{p^{\prime}}\right]\left[1+f_{k^{\prime}}\right]-f_{p^{\prime}} f_{k^{\prime}}\left[1+f_{p}\right]\left[1+f_{k^{\prime}}\right]\right\}, \\
\mathcal{C}_{1 \leftrightarrow 2}[f](p)= & \frac{(2 \pi)^{3}}{2|p|^{2}} \int_{0}^{\infty} d p^{\prime} d k^{\prime} \gamma_{p^{\prime}, k^{\prime}}^{p}\left(m, T_{*}\right)\left\{f_{p}\left[1+f_{p^{\prime}}\right]\left[1+f_{k^{\prime}}\right]-f_{p^{\prime}} f_{k^{\prime}}\left[1+f_{p}\right]\right\} \delta\left(p-p^{\prime}-k^{\prime}\right) \\
& +\frac{(2 \pi)^{3}}{|p|^{2}} \int_{0}^{\infty} d p^{\prime} d k \gamma_{p, k}^{p^{\prime}}\left(m, T_{*}\right) \delta\left(p+k-p^{\prime}\right)\left\{f_{p} f_{k}\left[1+f_{p^{\prime}}\right]-f_{p^{\prime}}\left[1+f_{p}\right]\left[1+f_{k}\right]\right\} .
\end{aligned}
$$

(Our matrix element is related to that of Ref. [15] by $|\mathcal{M}|^{2}=\sum_{b c d}\left|\mathcal{M}_{c d}^{a b}\right|^{2} / \nu, f=f_{a}$, and $\gamma=\gamma_{g g}^{g} / \nu$, where $\nu$ is the number of color and spin degrees of freedom. $\int_{\mathbf{p}} \equiv \int d^{3} p /(2 \pi)^{3}$.)

The effective matrix elements corresponding to elastic scattering $\left(|\mathcal{M}|^{2}\right)$ and collinear splitting $(\gamma)$ have been discussed in detail in Refs. $[12,15,18]$. The elastic collision term includes effects of screening appearing in the leading order by consistently regulating the Coulombic divergence in $t$ and $u$ channels at the screening scale $m$. The splitting kernel includes the effects of Landau-Pomeranchuk-Migdal (LPM) suppression [16,19], which regulates collinear divergences and depends on $m$ and an effective temperature

$$
T_{*}=\frac{1}{2} \int_{\mathbf{p}} f(p)[1+f(p)] / \int_{\mathbf{p}} \frac{f(p)}{|p|}
$$

that is self-consistently solved (along with $m$ ) during the simulation. The effective theory contains no free parameters besides the coupling constant $\lambda$. Our numerical implementation is the discrete- $p$ method of Ref. [12].

Overoccupied initial conditions.-We now determine the thermalization time of a system whose initial conditions are highly overoccupied. As discussed in the introduction, previous studies have established that overoccupied systems relax to a fixed point solution in the classical regime before thermalizing [7-14]. Hence, we take this solution as our initial condition in the following. In Ref. [12], the form of the fixed point solution at times $\tau_{\text {init }} \ll t \ll \tau_{\text {therm }}$ has been parametrized as $f(p)=\left(Q_{0} t\right)^{-4 / 7} \lambda^{-1} \tilde{f}(\tilde{p})$ with $\tilde{p}=\left(p / Q_{0}\right)\left(Q_{0} t\right)^{-1 / 7}$, and

$$
\tilde{f}(\tilde{p})=\frac{1}{\tilde{p}}\left(0.22 e^{-13.3 \tilde{p}}+2.0 e^{-0.92 \tilde{p}^{2}}\right),
$$

and $Q_{0}^{4}=2 \pi^{2} \lambda \int_{\mathbf{p}} p f(p)$. This scaling form is expected to be valid as long as the typical momentum scale is small compared to the thermal scale $\langle|p|\rangle \ll T$, corresponding to $f\left(p_{\text {max }}\right) \gg 1$ and $t \ll \tau_{\text {therm }}$. If $Q \ll T$, then $\tau_{\text {init }}$ and $\tau_{\text {therm }}$ are parametrically separated, and therefore the system will reside in the fixed point for a parametrically long time. Therefore, we can take the fixed point solution as our initial condition at some time $t_{0} \ll \tau_{\text {therm }}$ without loss of generality.

Figure 1 displays the time evolution of the distribution function. The dotted lines are from a simulation in the 


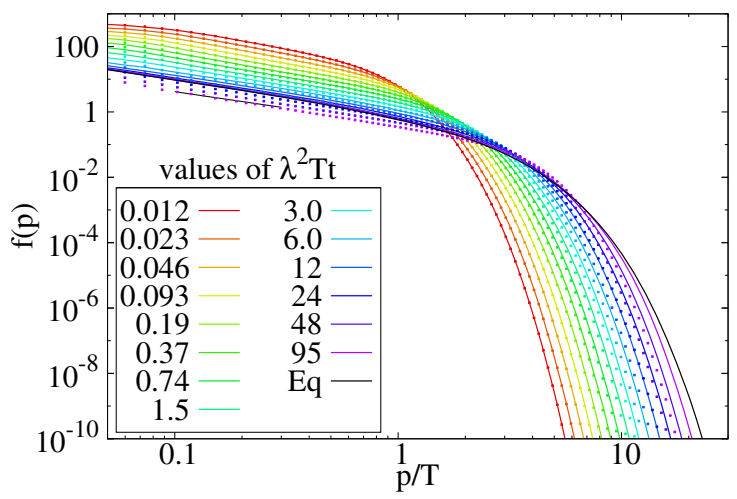

FIG. 1 (color online). Evolution of $f(p)$ towards the equilibrium distribution with overoccupied initial conditions and $\lambda=0.01$. The system starts from the scaling form of Eq. (7) at time $t_{0} \ll t_{\text {therm }}$ and relaxes towards the equilibrium distribution denoted by the black line. Even at late times, the ultraviolet tail of the distribution deviates from the thermal form. The full quantum evolution is compared to the classical approximation well described by Eq. (7) (dotted lines).

classical approximation. [The classical approximation is taken by including only the highest power of $f$ 's in the integrals of the right-hand side of Eq. (5).] In the classical approximation, there is no scale where the cascade would stop, and the cascade continues to move towards the UV ad infinitum as described by Eq. (7). The solid lines correspond to a simulation with the same initial condition, but now without making the classical approximation. In this case, the cascade ends to form the equilibrium distribution when the typical occupancies reach $f \sim 1$ when $\langle|p|\rangle \sim T$.

The time evolution of the various relevant moments of the distribution function are shown in Fig. 2. At times $t \ll \tau_{\text {therm }}$ the moments are well described by power laws predicted in Ref. [13] (dashed orange lines in the figure), but when $t \sim \tau_{\text {therm }}$ they smoothly approach their thermal values (red dashed lines). We repeated the simulation with several $\lambda=0.1-10$. We find that the $\lambda$ dependence is well accounted for by a rescaling of time, $\lambda^{2} T t\left(1+C_{2} \log \lambda^{-1}\right)$ with $C_{2}=0.12$. The logarithmic dependence in $\lambda$ arises from an IR divergence in momentum diffusion caused by soft elastic collisions in the $m \rightarrow 0$ limit. We show our estimate for the thermalization time of Eq. (2) with a vertical line, which we have extracted by using the definition of Eq. (1). It is noteworthy, however, that the various moments shown in the figure reach $\sim 50 \%$ of their thermal values within a much faster time scale $\sim 0.01 t_{\text {eq }}$.

Bottom-up thermalization.-For the underoccupied system the choice of initial conditions will affect the system until it is thermalized, and therefore we do not experience similar universal behavior as in the overoccupied case. Here, we will proceed by first choosing a specific set of initial conditions. For maximally different initial conditions, we use either a step function or a Gaussian profile for the distribution function

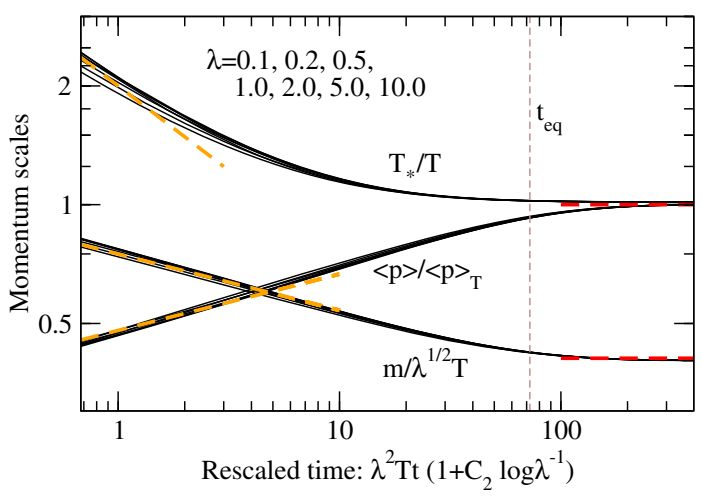

FIG. 2 (color online). Evolution of various moments $\left(T_{*}, m\right.$, and $\langle|p|\rangle)$ as a function of rescaled time with overoccupied initial conditions. The different lines correspond to different values of $\lambda=0.1-10$. The orange dashed lines correspond to the classical limit, and the red dashed lines are the thermal values. The vertical line is the thermalization time of Eq. (2).

$f_{\text {step }}(p) \propto \Theta\left(Q_{s}-p\right), \quad f_{g}(p) \propto \exp \left[-\frac{\left(Q_{s}-p\right)^{2}}{\left(Q_{s} / 10\right)^{2}}\right]$

and fix the constant of proportionality so that the final temperature $T$ is in each case the same. Our simulation parameters are given in Table I.

The full time evolution of a representative initial condition from $t=0$ to thermalization is shown in Fig. 3. Two distinct stages are seen in the evolution: at early times (solid lines), the initial hard particles at the scale $Q$ emit soft radiation leading to a buildup of a soft thermal bath. At late times (black dashed lines), the hard particles undergo radiational breakup and become part of the thermal bath (red dashed line).

At early times, there are three clearly separated structures visible in the spectrum. (a) The hard particles residing at the initial scale $Q$ have not had time to scatter and remain very close to the initial condition. (b) The hard particles emit LPM suppressed radiation with a characteristic $f \propto p^{-7 / 2}$ spectrum. (c) In the far IR where $f \gtrsim 1$, reinteractions are fast enough to bring soft modes close to a thermal form.

The underoccupied system finally thermalizes through a radiative breakup. Once the hard particles have had time to undergo a single democratic splitting, i.e., splitting to

TABLE I. Table of simulation parameters for the underoccupied system. The initial particle number density is given in units of the thermal density $n_{T}$. The last column refers to the initial condition as given by Eq. (8).

\begin{tabular}{lccccccc}
\hline \hline Run & $Q / T$ & $\lambda$ & Init & Run & $Q / T$ & $\lambda$ & Init \\
\hline 1 & 202.5 & 0.1 & $\mathrm{~g}$ & 4 & 155.1 & 0.1 & Step \\
2 & 404.9 & 0.1 & $\mathrm{~g}$ & 5 & 310.0 & 0.1 & Step \\
3 & 809.8 & 0.1 & $\mathrm{~g}$ & 6 & 620.0 & 0.1 & Step \\
7 & 155.1 & 1.0 & Step & 9 & 310.0 & 1.0 & Step \\
8 & 155.1 & 10.0 & Step & 10 & 310.0 & 10.0 & Step \\
\hline \hline
\end{tabular}




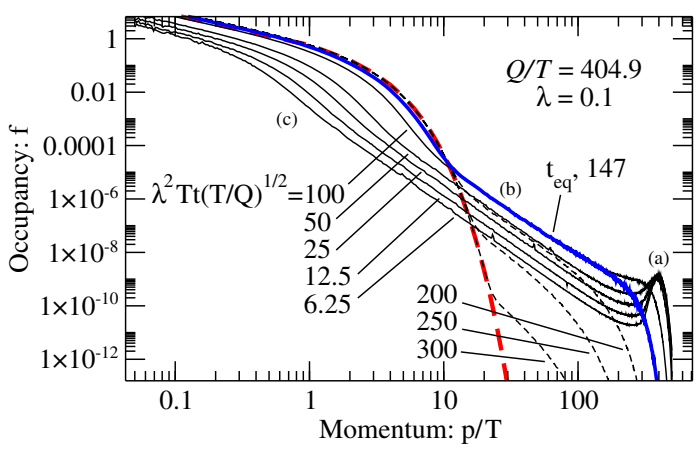

FIG. 3 (color online). The time evolution for an underoccupied system (run 2). At early times, soft radiation emitted by the hard particles creates a thermal bath (solid lines). When the hard particles have had time to undergo a democratic splitting to daughters of comparable energy, the hard particles undergo a radiational breakup (dashed lines) and become part of the thermalized bath. The solid blue line corresponds to the thermalization time of Eq. (3), while the red dashed line is the equilibrium distribution. The structures (a)-(c) discussed in the text are indicated in the figure for convenience.

daughters with comparable momenta, the resulting daughters will split again in a time scale that is faster than the initial splitting $[5,20]$. The daughters undergo successive resplittings until they have deposited their energy into the thermal bath (dashed lines in Fig. 3). Parametrically, the rate for the first hard splitting and therefore for the cascade is

$$
\tau_{\text {split }} \sim(Q / T)^{1 / 2} \times 1 / \lambda^{2} T
$$

up to logarithmic corrections [13]. As in the case of the overoccupied system, we observe that the dependence of the various moments of distribution function on $\lambda$ can be accounted for by a multiplicative rescaling of the time variable, including a logarithm $\lambda^{2} T t\left(1+C_{3} \log \lambda^{-1}\right)$ with $C_{3} \approx 0.037$. We furthermore find that most of the dependence on the initial condition can be accounted for by rescaling the time by $(T / Q)^{1 / 2}$ as expected from the parametric estimate for the thermalization time.

After the rescaling, $m, T_{*}$, and $\langle|p|\rangle$ show only weak dependence on the initial conditions which is well described by a logarithmic delay of the thermalization as a function of $Q / T$ : we find a satisfactory collapse of the data at late times when plotted as a function of

$$
t_{\text {rescaled }}=\lambda^{2} T t\left(1+C_{3} \log \lambda^{-1}\right)(T / Q)^{1 / 2}-C_{4} \log (Q / T)
$$

with $C_{4} \approx 21$ as seen in Fig. 4 . We extract the thermalization time from exponential decay of $\langle|p|\rangle-\langle|p|\rangle_{T}$ as a function of the rescaled time variable giving $\left.t_{\text {eq }}\right|_{\text {rescaled }} \approx 34$ (vertical line in Fig. 4). Converting this back to unscaled time leads to the thermalization time of Eq. (3). We observe, however, that high moments (such as $\left\langle p^{2}\right\rangle$ ) show

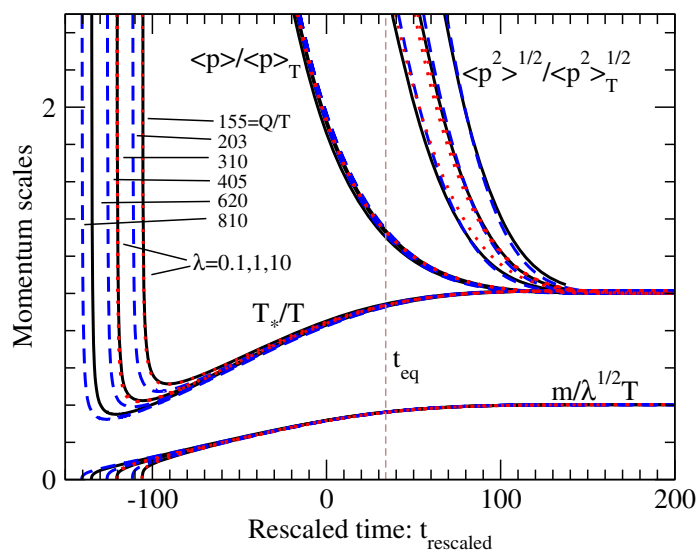

FIG. 4 (color online). Evolution of various moments of the distribution function from the simulations of Table I as a function of rescaled time, Eq. (10). The dashed blue lines correspond to Gaussian initial conditions with $\lambda=0.1$, whereas solid black ( $\lambda=0.1)$ and dotted red lines $(\lambda=1,10$, on top of black lines) have step-cutoff initial conditions. $Q$ decreases from left to right. The dashed vertical line is the thermalization time as given by Eq. (2).

sensitivity to the initial conditions arising from increased sensitivity to harder parts of the distribution function, and the dependence on the initial conditions is not removed by a simple rescaling of time. Unlike in the overoccupied case, the system (in particular, the high moments) differs significantly from the equilibrium system before the thermalization time.

Summary and conclusions. - In this Letter, we have for the first time simulated the time evolution of weakly coupled non-Abelian plasmas from far-from-equilibrium initial conditions to thermal equilibrium. We performed the simulation in a framework of an effective kinetic theory that has no free parameters and gives an accurate description of the gauge theory in the combined limit of $\lambda f \ll 1$ and $\lambda \ll 1[15]$.

How soon the system can be considered thermal is observable dependent. Here we have chosen to define the thermalization time through the relaxation of mean momentum, but defining the thermalization time through the relaxation of $m^{2}$ or $T_{*}$ results in values compatible with our definition.

Our results from nonexpanding simulations can be applied to elevate the parametric weak coupling estimate of thermalization time in heavy-ion collisions of Baier, Mueller, Schiff, and Son to a numerical one [5]. Their estimate can be quickly derived by assuming that as a result of longitudinal expansion the energy density of the system falls as a function of (proper) time, $\epsilon \sim\left(Q^{3} / \lambda t\right)$; so the target temperature falls as $T^{4} \sim Q^{3} / \lambda t$. Inserting this into the parametric estimate of the thermalization time of an underoccupied system of Eq. (9) and solving for the thermalization time by equating $\tau_{\mathrm{eq}} \sim t$ gives the estimate of Ref. [5]: $Q t \sim \alpha_{s}^{-13 / 5}$. Replacing the parametric estimate for the thermalization time by Eq. (3), assuming that the 
energy density is given by $\epsilon \approx 1.5 d_{A} Q^{4} / \pi \lambda(Q t)$ (with $d_{A}=8$ ) [21], choosing $\lambda=11$ corresponding to $\alpha_{s} \approx 0.3$, and solving the thermalization time self-consistently gives $Q t_{\text {eq }} \approx 1.5$. (We derive the energy density from the product the gluon multiplicity [Eq. (14) of Ref. [21]] and mean transverse momentum of the gluons which we read from Fig. 6 of Ref. [21]: $\epsilon=\left\langle p_{T}\right\rangle \times\left(d N_{\text {init.g }} / d^{2} \mathbf{x}_{T} d y\right) / \tau$. $)$ Smaller values of $\alpha_{s}$ lead to slower thermalization: for $\alpha_{s}=0.2$ we get $Q t_{\text {eq }} \approx 4.0$. Varying the estimate for the energy density by a factor of 2 gives $Q t_{\mathrm{eq}} \lesssim 2.5\left(Q t_{\mathrm{eq}} \lesssim 8\right.$ for $\alpha_{s}=0.2$ ), whereas multiplying $\epsilon$ by $(Q t)^{-1 / 3}$ to estimate an upper bound for redshift effects gives $Q t_{\text {eq }} \lesssim 4$ (for $\alpha=0.3$ ). For $Q \sim 2 \mathrm{GeV}$ these values correspond to an early thermalization time of $t_{\mathrm{eq}} \approx 0.2-1 \mathrm{fm} / c$. Our conclusion is that rapid thermalization is not in contradiction with a weak coupling picture.

The estimate for the thermalization time can be further improved by taking into account the angular dependence of the distribution function. However, the dominant interaction causing the radiative breakup of the hard particles is against the thermal (and hence nearly isotropic [6]) background, and therefore we believe that introducing angular dependence to the EKT will not change the result qualitatively. We also expect minor corrections from including fermions in the EKT. We leave these improvements for a forthcoming publication.

The authors thank Juergen Berges, Simon Caron-Huot, Mathias Garny, François Gelis, Liam Keegan, Tuomas Lappi, Aleksi Vuorinen, and Urs Wiedemann for useful discussions. The authors are indebted to Guy Moore for support, advice, and collaboration in the development of some of the tools used in this study. This work was supported in part by the Canadian Natural Sciences and Engineering Research Council (NSERC) and Dr. and Mrs. Milton Leong Fellowship.

[1] L. Kofman, A. D. Linde, and A. A. Starobinsky, Phys. Rev. Lett. 73, 3195 (1994); J. Garcia-Bellido, D. G. Figueroa, and J. Rubio, Phys. Rev. D 79, 063531 (2009); K. Enqvist, S. Nurmi, and S. Rusak, arXiv:1404.3631.
[2] J. M. Cline, arXiv:hep-ph/0609145.

[3] E. Iancu, A. Leonidov, and L. McLerran, arXiv:hep-ph/ 0202270; E. Iancu and R. Venugopalan, arXiv:hep-ph/ 0303204; F. Gelis, T. Lappi, and R. Venugopalan, Int. J. Mod. Phys. E 16, 2595 (2007); F. Gelis, E. Iancu, J. JalilianMarian, and R. Venugopalan, Annu. Rev. Nucl. Part. Sci. 60, 463 (2010).

[4] K. Harigaya, M. Kawasaki, K. Mukaida, and M. Yamada, Phys. Rev. D 89, 083532 (2014); K. Mukaida and K. Nakayama, J. Cosmol. Astropart. Phys. 03 (2013) 002; K. Harigaya and K. Mukaida, J. High Energy Phys. 05 (2014) 006.

[5] R. Baier, A. H. Mueller, D. Schiff, and D. T. Son, Phys. Lett. B 502, 51 (2001).

[6] A. Kurkela and G. D. Moore, J. High Energy Phys. 11 (2011) 120.

[7] J. Berges, S. Scheffler, and D. Sexty, Phys. Lett. B 681, 362 (2009).

[8] J. Berges, S. Schlichting, and D. Sexty, Phys. Rev. D 86, 074006 (2012).

[9] S. Schlichting, Phys. Rev. D 86, 065008 (2012).

[10] A. Kurkela and G. D. Moore, Phys. Rev. D 86, 056008 (2012).

[11] J. Berges, K. Boguslavski, S. Schlichting, and R. Venugopalan, Phys. Rev. D 89, 114007 (2014).

[12] M. C. Abraao York, A. Kurkela, E. Lu, and G. D. Moore, Phys. Rev. D 89, 074036 (2014).

[13] A. Kurkela and G. D. Moore, J. High Energy Phys. 12 (2011) 044.

[14] J.-P. Blaizot, F. Gelis, J.-F. Liao, L. McLerran, and R. Venugopalan, Nucl. Phys. A873, 68 (2012).

[15] P. B. Arnold, G. D. Moore, and L. G. Yaffe, J. High Energy Phys. 01 (2003) 030.

[16] R. Baier, Y. L. Dokshitzer, A. H. Mueller, S. Peigne, and D. Schiff, Nucl. Phys. B483, 291 (1997); 484, 265 (1997); B. G. Zakharov, JETP Lett. 63, 952 (1996).

[17] P. B. Arnold, S. Cantrell, and W. Xiao, Phys. Rev. D 81, 045017 (2010).

[18] P. B. Arnold and C. Dogan, Phys. Rev. D 78, 065008 (2008).

[19] L. D. Landau and I. Pomeranchuk, Dokl. Akad. Nauk Ser. Fiz. 92, 535 (1953); A. B. Migdal, Phys. Rev. 103, 1811 (1956).

[20] J.-P. Blaizot, E. Iancu, and Y. Mehtar-Tani, Phys. Rev. Lett. 111, 052001 (2013)

[21] T. Lappi, Phys. Lett. B 703, 325 (2011). 